\title{
Functional endoscopic sinus surgery (FESS): Is it always a safe procedure?
}

\author{
Enrico M Amadei* \\ Department of Otorhinolaryngology and Audiology, Infermi Hospital, Rimini, Italy
}

\begin{abstract}
Functional Endoscopic Sinus Surgery (FESS) is an easy, fast and effective surgical procedure for treating rhinosinusitis that is not responsive to medical therapy. We never had major post-FESS complications up to 6 months ago. Therefore, we wanted to compare our results with those of Literature. During the period 2013-2018 we performed 589 FESS interventions. We had a percentage of minor complications similar to reported in the Literature (5-10\%), and a lower incidence of major complications $(0.34 \%)$. We therefore look in detail at our cases of major complications, with the support of pre- and post-operative imaging.

We believe that it is essential to carefully study preoperative imaging. Besides operating in a standardized manner, despite the variation of anatomical features, it is the best way to prevent complications of a FESS. In a department of Otorhinolaryngology without a high-volume series like ours, the complication percentages derived from FESS are low and comparable with those of Literature. This datum is very useful, as in Literature there are numerous huge series, gathered in major world Hospitals, while the data collected in non-high-volume Centres are scarce, even if here large number of FESS is performed every year.
\end{abstract}

\section{Introduction}

In our Operating Unit we performed 589 Functional Endoscopic Sinus Surgeries (FESS) during the period 2013-2018. This kind of surgery is indicated for the treatment of chronic hyperplastic rhinosinusitis that is not responsive to medical therapy and to removing nasal polyps [1]. The purpose is to take away the hypertrophic mucosa and to enlarge the natural drainage pathways that connect the paranasal sinuses with nasal cavities. FESS is generally preferred over open techniques, because it is faster, less aggressive for the patient, and with shorter healing times.

Typically, this type of surgery at our Hospital lasts about 1.5-2 hours, including the times of falling asleep and awakening of general anaesthesia. We administer to the Patient $(\mathrm{Pt})$ an oral steroid therapy the week before surgery. We do not perform any pre-operative antibiotic prophylaxis and no routine post-operative antibiotic therapy. We never interrupt the habitual antiplatelet therapy, if any, and we administer subcutaneous heparin for antithrombotic prophylaxis if there are risk factors, for a week after surgery. Upon awakening, the patient has bilateral intranasal packing, which will be removed on the first postoperative day. Hospital discharge occurs the first day after surgery and it takes place approximately one hour from the medication and nasal packing removal.

Since many years FESS is no longer considered an experimental surgery, and it is now established in the routine surgery of many Otorhinolaryngologists. FESS is considered a safe technique with few risks. But we must remember that the danger is always around the corner. In fact, as we often say with simple words to the Pt at the time of informed consent, "the nose is positioned between the eyes and below the brain". So, the complications can concern the orbital region, above all with ecchymosis and emphysema, following the rupture of the lamina papyracea. Complications may include the intracranial region, with pneumocephalus, meningitis or abscess, following a fistula of the anterior cranial base. Complications can eventually result from bleeding or intranasal infections.
Surgical complications are generally divided into major and minor [2]. Many scientific papers now indicate only the percentages of major complications $[3,4]$, reporting for example the incidence of orbital injury, CSF leak, severe haemorrhage and toxic shock syndrome. In fact, a transient periorbital ecchymosis is often not considered a significant problem by many patients undergoing FESS.

We never had major post-FESS complications up to 6 months ago. Therefore, we wanted to compare our results with those of Literature.

\section{Materials and results}

This paper does not violate ethical principles, as we have carried out all the FESS interventions to the maximum of our possibilities. Besides this paper does not violate privacy, because all data are anonymous, and identifiable patients gave their consent to this study.

We performed 589 Functional Endoscopic Sinus Surgeries (FESS) during the period 2013-2018. Our percentages of minor complications are comparable to those of Literature [2], while our major complications were rarer. We never had significant orbital injury, severe haemorrhage or toxic shock syndrome. We had two cases of CSF leak, both happened in the last few months: a CSF fistula, complicated with pneumocephalus and a CSF leak, associated with acute meningitis.

${ }^{*}$ Correspondence to: Enrico Maria Amadei, Department of Otorhinolaryngology and Audiology, Infermi Hospital, Rimini, Italy, Tel: +39 3283625727; E-mail: enricomaria.amadei@libero.it

Key words: FESS, endoscopic sinus surgery, complications, CSF leak, pneumocephalus, meningitis

Received: February 25, 2019; Accepted: March 15, 2019; Published: March 19, 2019 


\section{Case 1}

We describe the case of a 37-year-old man with a bilateral chronic hyperplastic rhinosinusitis (Figure 1). Nasal polyposis was not massive, and it was only partially occluding the paranasal sinuses. However, the Pt complained of hyposmia and severe nasal congestion, not responsive to medical therapy with nasal washes and cortisone spray. So, we performed a FESS, without immediate complications.

The day after surgery we removed nasal packing and suddenly the Pt reported a strong transient headache. After a few minutes the Pt felt good again, and we sent him home. However, the headache returned in the afternoon and it persisted the following hours. It was intense and exacerbated by the efforts. So, the next day we performed an urgent CT scan without contrast, and we found a moderate pneumocephalus, without the leakage of cerebrospinal fluid (CSF) from the nose (Figures 2 and 3 ).

Therefore, we re-admitted the Pt and we subjected him to parenteral antibiotic therapy with amoxicillin plus clavulanic acid three times a day for 10 days. We decided not to perform a new surgical operation on him, as there was never nasal loss of CSF. We monitored the Pt over the days, performing two additional control CTs: the first one 2 days after admission and the second one 10 days later. At last Imaging the pneumocephalus was completely reabsorbed and the headache resolved (Figure 4). Pt had no infectious complications or chronic headache at a 5-months follow-up.

\section{Case 2}

We report the case of a 42 -year-old man with a hypertrophy of the inferior nasal turbinates and a left nasal respiratory difficulty. At CT scan a neoformation of the left middle turbinate was found (Figure 5). An inverted papilloma was diagnosed on biopsy. So, we performed an endoscopic surgery in the left nasal fossa, to remove the lesion.

The following day the Pt began to appear disoriented, deaf and with a marked hyperthermia. Acute meningitis was diagnosed at the neurological evaluation. A brain CT scan first and a subsequent

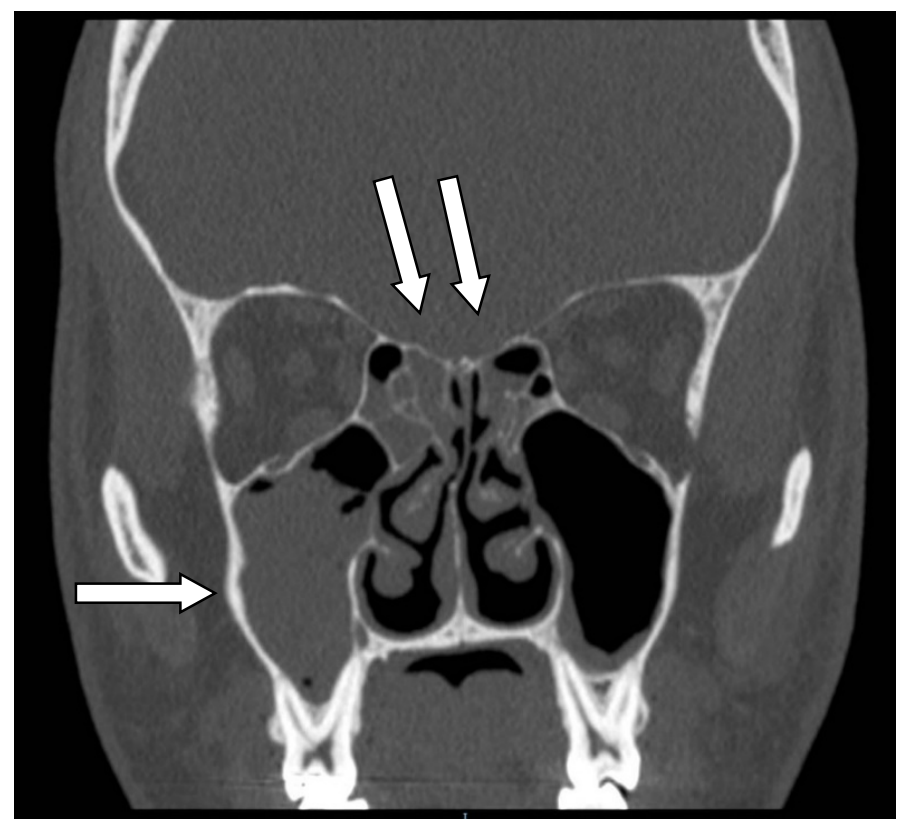

Figure 1. First Pt. Pre-surgery maxillofacial, CT: we can see right maxillary sinusitis (see single arrow) and ethmoidal bilateral sinusitis (see double arrows).

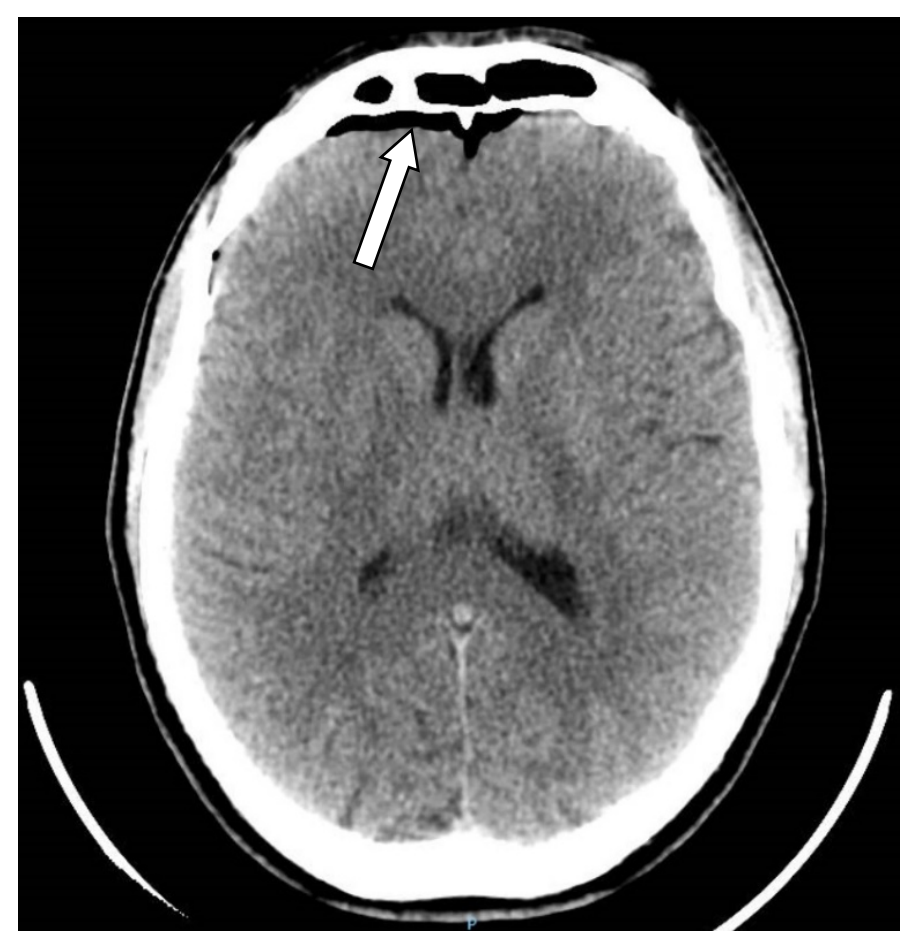

Figure 2. First Pt. Post-surgery brain, CT: frontal pneumocephalus (see arrow).

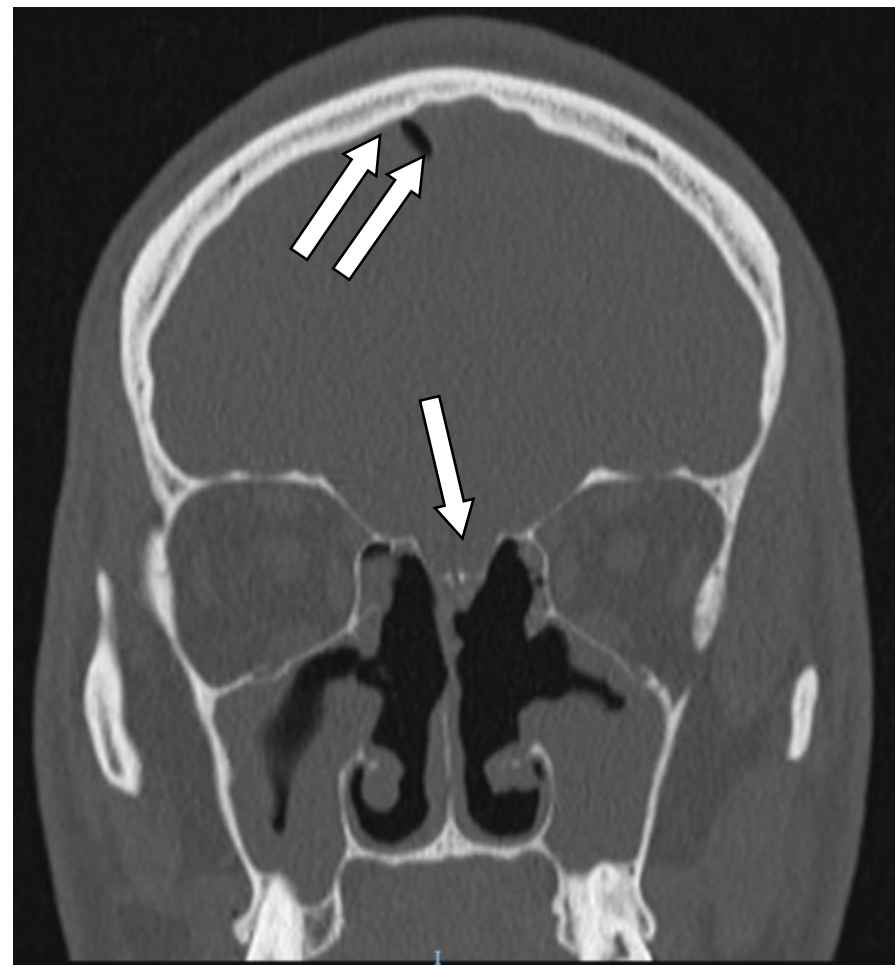

Figure 3. First Pt. Post-surgery maxillofacial, CT: dehiscence of cribriform plate (see single arrow); pneumocephalus of apex (see double arrows).

maxillofacial CT scan without contrast found a pneumocephalus and a fistula of the anterior cranial base (Figures 6 and 7): a CSF leak of about $4 \mathrm{~mm}$ was observed between the cribriform plate and the posterior medial margin of the left frontal sinus.

The Pt was then transferred to intensive care. Here pneumococcal meningitis was diagnosed by means of the culture examination on 


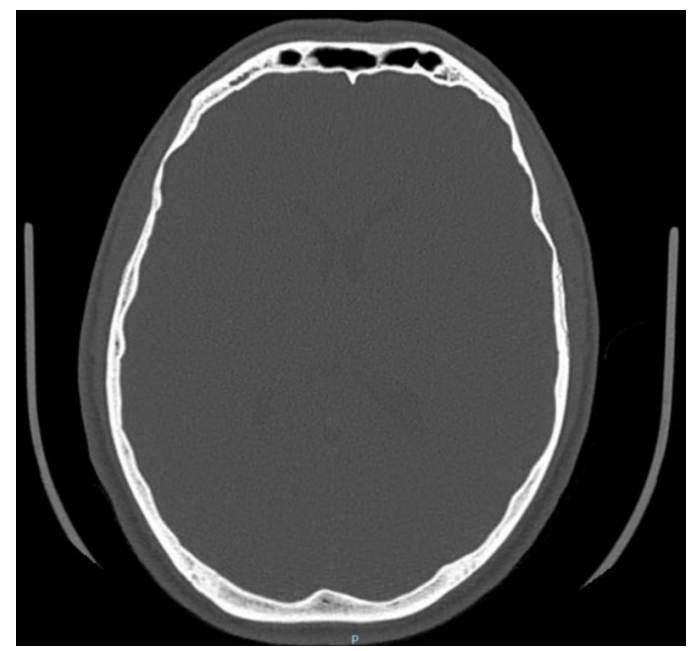

Figure 4. First Pt. Post-surgery maxillofacial, CT: resolution of pneumocephalus.

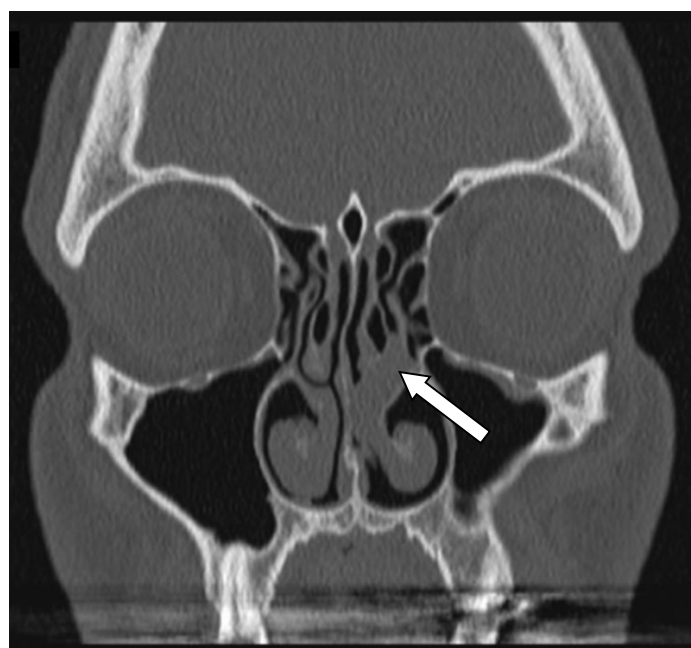

Figure 5. Second Pt. Pre-surgery maxillofacial, CT: we can see bilateral hypertrophic inferior turbinates and a polyp at the left middle meatus (see arrow)

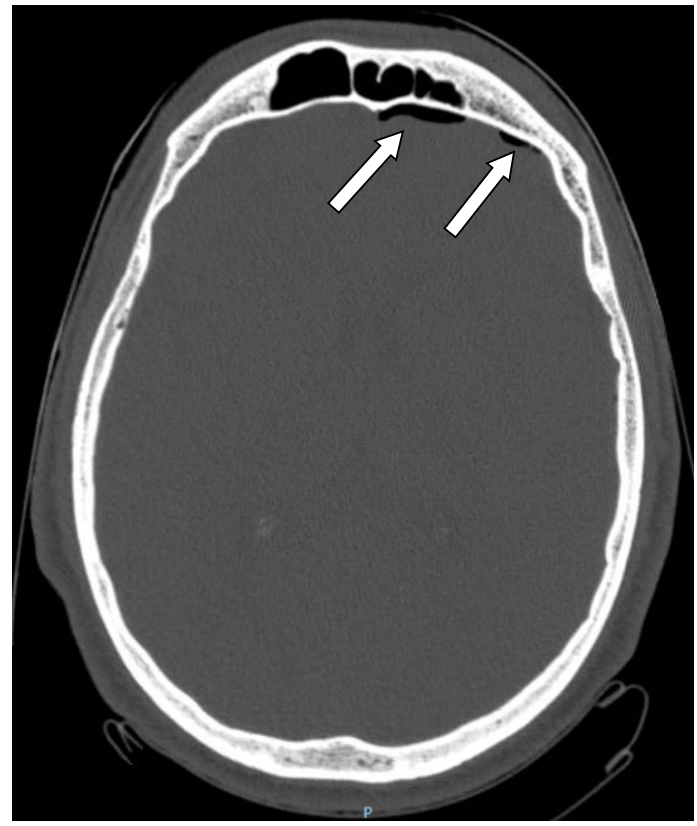

Figure 6. Second Pt. Post-surgery maxillofacial, CT: left frontal pneumocephalus (see arrows). 


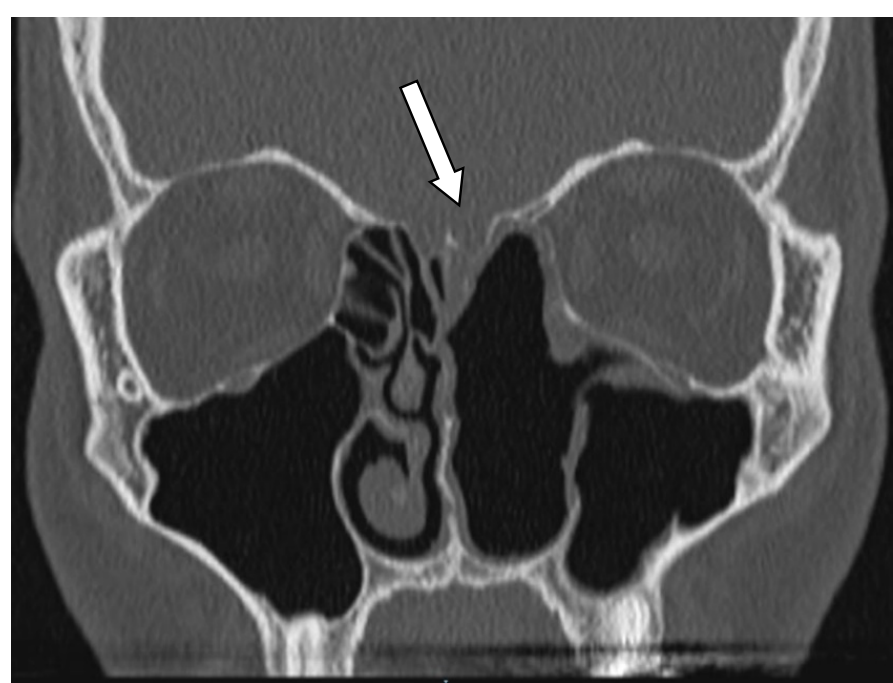

Figure 7. Second Pt. Post-surgery maxillofacial, CT: we can see the removal of all left turbinates and a left parasagittal dehiscence of cribriform plate (see arrow).

the cerebral liquor, after a lumbar puncture. Therapy with parenteral ceftriaxone and levetiracetam (anticonvulsant drug) two times a day was performed. After 11 days we stopped the ceftriaxone, due to the onset of a herpetic rash in the Pt's face. We then administered moxifloxacin $400 \mathrm{mg} 1$ tablet per os a day for a further 15 days.

Twenty five days after the first surgery, the Pt was subjected to endoscopic closure of the CSF leak, with a good functional result. No infective relapse occurred two months after the last surgery. No neurological sequelae remained. No further CTs were performed.

\section{Discussion}

FESS is universally considered a safe technique with few risks. In the Literature there is an incidence of $5 \%$ for minor complications and of $0.5-1 \%$ for major complications [2]. The risks can increase in cases of $\mathrm{Pt}$ with pluri morbidity of revision surgery, or of a very advanced nasal pathology. We collected a series of 589 Pts, submitted to FESS at our Surgical Unit in the period 2013-2018.

In our casuistry we have a rate of ecchymosis of the eyelids with or without orbital emphysema of $10 \%$, nasal synechiae of $5 \%$, nasal bleeding of $5 \%$. Besides we had a single case of CSF fistula, complicated with pneumocephalus, and a single case of CSF leak associated with acute pneumococcal meningitis.

We have an incidence of minor complications similar to Literature $(5-10 \%)[2,5,6]$. We treat ecchymosis of the eyelids with an early removal of nasal packing, even an hour after surgery. We have never had significant orbital damages. Regarding nasal synechiae, we leave them, if Pt does not feel bothered. Otherwise we dissect them under local or general anaesthesia, positioning a tampon or a Teflon plaque to keep the nasal structures spaced until healing, in order to prevent readhesion. If nasal bleeding occurs after surgery, we perform an electric caustication of bleeding vessels or we place a new nasal packing.

Major complications are a bit less frequent than what is reported in Literature: ours are $0.34 \%$, compared to $0.5-1 \%$ of Hosemann et al. [2], $0.41 \%$ according to Krings et al. [3], 0.50\% according to Suzuki et al. [4], 2.4\% according to Stankiewicz et al. [7]. The reason probably lies in the fact that we have not a large series and we are not a secondlevel reference Centre for nasosinus pathology. We typically execute a "virgin patient" surgery, with only a minority of revision surgery cases, and we do not perform extensive surgery on the anterior cranial base. This explains why in our casuistry there are no cases of encephaloceles, sight loss or cerebral abscesses, and the rarity of CSF fistulae.

Krings et al. [3] wrote that complications following revision FESS are similar to primary cases $(0.46 \%$ and $0.36 \%$ respectively). But we believe that performing a surgical procedure in a secondary patient is much riskier, especially if the first surgery was performed elsewhere. Often the operating steps may not be well described, or important anatomical landmarks may be lost. This according to other Authors [2,7]

Suzuki et al. said that the extent of surgery did not significantly affect the overall complication rate. We present cases that are only minimally comparable to theirs (500 against 50000!). Nevertheless, we think that for a surgeon who is not only dedicated to FESS, and who has not such a vast experience, performing a more extensive FESS is certainly a more demanding and riskier act. This is in the agreement written by Others [2,7].

We always require a pre-operative maxillofacial CT scan without contrast for the Pt. In the event of a unilateral lesion, we also carry out a MRI. We believe that carefully studying the Imaging before surgery allows recognizing anatomical variants, to predict and to prevent possible complications [8]

About the first Pt of this paper, our mistake was to push ourselves too high, going to open the anterior cranial base. Probably we made this while looking for the front-ethmoidal left recess. At the maxillofacial CT we detected a bone discontinuity that affects the ethmoidal roof, with greater evidence in the left parasagittal region (Figure 3 ). We were lucky, because there was no CSF leakage from the fistula. This means that we did a defect in the skull base, thus favouring the onset of the pneumocephalus, but we have not damaged the dura. Pt healed by observation and antibiotic therapy only.

In the second case our error was to fracture the root of the left middle turbinate, without cutting it with caution. In this way we damaged the cribriform plate and the overlying meninges (Figures 5-7). We know that bacterial meningitis often occurs slowly within a few days: on the contrary instead in this case the hyperthermia and the obnubilation of the patient had suddenly arisen. So, we had to contact the neurologist and the anaesthetist at less than 24 hours from the surgical intervention. So, we first treated the acute meningitis, then after about 1 month we repaired the CSF leak. We used the contralateral septal mucoperichondrial fascia, with an overlay technique, after an accurate removal of the nasal mucosa and a milling of the bony asperities surrounding the fistula.

The limit of this study is its small number of cases, since the low incidence of complications is more significant only with large cases. However, the small casuistry is also the strength and the peculiarity of this study: we work in a minor Centre and we had never had major complications until 6 months ago. We therefore wanted to compare our results with those reported in Literature. We believe that this attitude is an unequivocal sign of seriousness, with the aim of improving our results more and more.

\section{Conclusions}

We believe that FESS is a safe, effective and fast procedure. In our series the incidence of minor and major complications is $5-10 \%$ and $0.34 \%$ respectively.

We think that performing a revision FESS is more difficult and time demanding than a primary FESS, due to altered anatomy and 
scarring. In our case series the worst complications occurred in 2 apparently simple cases, and this leads us to emphasize the fact that every intervention always involves risks, and that the surgeon must operate in an always careful, precise and standardized way, to avoid incorrect manoeuvres and unpleasant incidents.

From the Literature we already knew that the incidence of major complications was rare $(0.5 \%)$, but there were no papers with "local" casuistry of about 100 FESS interventions per year. These numbers can be reached in the vast majority of ENT Surgical Centres and therefore, in our opinion, are more indicative of the risks to which the "standard Pt" can go.

We can say that even in a department of Otorhinolaryngology without a high-volume series like ours, the complication percentages derived from FESS are low and comparable with the main international Centres.

Another element to remember is that the surgeon who approaches FESS must be able to recognize any CSF leak promptly, better if during the first surgery, and must be able to repair it. Carrying out this procedure makes it possible to remedy major complications, resulting from a bone dehiscence, and it often also allows them to be prevented (pneumocephalus and meningitis).

\section{References}

1. Becker SS (2009) Surgical Management of Polyps in the Treatment of Nasal Airway Obstruction. Otolaryngol Clin North Am 42: 377-385. [Crossref]

2. Hosemann W, Draf C (2013) Danger points, complications and medico-legal aspects in endoscopic sinus surgery. GMS Curr Top Otorhinolaryngol Head Neck Surg 12: Doc06. [Crossref]

3. Krings JG, Kallogjeri D, Wineland A, Nepple KG, Piccirillo JF, et al. (2014) Complications of Primary and Revision Functional Endoscopic Sinus Surgery for Chronic Rhinosinusitis. Laryngoscope 124: 838-845. [Crossref]

4. Suzuki S, Yasunaga H, Matsui H, Fushimi K, Kondo K, et al. (2015) Complication Rates After Functional Endoscopic Sinus Surgery: Analysis of 50,734 Japanese Patients. Laryngoscope 125: 1785-1791. [Crossref]

5. Dalziel K, Stein K, Round A, Garside R, Royle P (2006) Endoscopic sinus surgery for the excision of nasal polyps: A systematic review of safety and effectiveness. Am J Rhinol 20: 506-519. [Crossref]

6. Tan BK, Chandra RK (2010) Postoperative Prevention and Treatment of Complications After Sinus Surgery. Otolaryngol Clin North Am 43: 769-779. [Crossref]

7. Stankiewicz JA, Lal D, Connor M, Welch K (2011) Complications in endoscopic sinus surgery for chronic rhinosinusitis: a 25-year experience. Laryngoscope 121: 26842701. [Crossref]

8. Vemuri NV, Karanam LSP, Manchikanti V, Dandamudi S, Puvvada SK, et al. (2017) Imaging review of cerebrospinal fluid leaks. Indian J Radiol Imaging 27: 441-446. [Crossref]

Copyright: (C2019 Amadei EA. This is an open-access article distributed under the terms of the Creative Commons Attribution License, which permits unrestricted use, distribution, and reproduction in any medium, provided the original author and source are credited. 